\title{
AN OPTIMAL POINCARÉ-WIRTINGER INEQUALITY IN GAUSS SPACE
}

\author{
Barbara Brandolini, Francesco Chiacchio, Antoine Henrot and \\ Cristina Trombetti
}

\begin{abstract}
Let $\Omega$ be a smooth, convex, unbounded domain of $\mathbb{R}^{N}$. Denote by $\mu_{1}(\Omega)$ the first nontrivial Neumann eigenvalue of the Hermite operator in $\Omega$; we prove that $\mu_{1}(\Omega) \geq$ 1. The result is sharp since equality sign is achieved when $\Omega$ is a $N$-dimensional strip. Our estimate can be equivalently viewed as an optimal Poincaré-Wirtinger inequality for functions belonging to the weighted Sobolev space $H^{1}\left(\Omega, d \gamma_{N}\right)$, where $\gamma_{N}$ is the $N$-dimensional Gaussian measure.
\end{abstract}

\section{Introduction}

Let $\Omega$ be a convex domain of $\mathbb{R}^{N}(N \geq 2)$ and let us denote by $d \gamma_{N}$ the standard Gaussian measure in $\mathbb{R}^{N}$, that is

$$
d \gamma_{N}=\frac{1}{(2 \pi)^{N / 2}} e^{-\frac{|x|^{2}}{2}} d x .
$$

In [3] (see also [37] and [2]) the authors prove, among other things, that if $\Omega$ is a convex, bounded domain, the first nontrivial eigenvalue $\mu_{1}(\Omega)$ of the following problem:

$$
\begin{cases}-\operatorname{div}\left(\exp \left(-\frac{|x|^{2}}{2}\right) D u\right)=\mu \exp \left(-\frac{|x|^{2}}{2}\right) u & \text { in } \Omega \\ \frac{\partial u}{\partial \nu}=0 & \text { on } \partial \Omega\end{cases}
$$

satisfies

$$
\mu_{1}(\Omega) \geq \max \left\{1, \frac{1}{2}+\frac{\pi^{2}}{\operatorname{diam}(\Omega)^{2}}\right\} .
$$

Here $\nu$ stands for the outward normal to $\partial \Omega$ and $\operatorname{diam}(\Omega)$ for the diameter of $\Omega$. It is well known that $\mu_{1}(\Omega)$ can be characterized in the following variational way:

$$
\mu_{1}(\Omega)=\min \left\{\frac{\int_{\Omega}|D \psi|^{2} d \gamma_{N}}{\int_{\Omega} \psi^{2} d \gamma_{N}}: \psi \in H^{1}\left(\Omega, d \gamma_{N}\right) \backslash\{0\}, \int_{\Omega} \psi d \gamma_{N}=0\right\},
$$

where $H^{1}\left(\Omega, d \gamma_{N}\right)$ is the weighted Sobolev space defined as follows:

$$
H^{1}\left(\Omega, d \gamma_{N}\right)=\left\{u \in W_{\mathrm{loc}}^{1,1}(\Omega):(u,|D u|) \in L^{2}\left(\Omega, d \gamma_{N}\right) \times L^{2}\left(\Omega, d \gamma_{N}\right)\right\}
$$

Received by the editors September 28, 2012.

1991 Mathematics Subject Classification. 35B45; 35P15; 35J70.

Key words and phrases. Neumann eigenvalue, Hermite operator, sharp bounds. 
endowed with the norm

$$
\|u\|_{H^{1}\left(\Omega, d \gamma_{N}\right)}=\|u\|_{L^{2}\left(\Omega, d \gamma_{N}\right)}+\|D u\|_{L^{2}\left(\Omega, d \gamma_{N}\right)} .
$$

Incidentally note that the space $H^{1}\left(\Omega, d \gamma_{N}\right) \equiv H^{1}(\Omega)$ whenever $\Omega$ is a bounded domain.

Estimate (1.2) implies that, if $\Omega$ is a bounded, convex domain of $\mathbb{R}^{N}$, the following Poincaré-Wirtinger inequality holds:

$$
\int_{\Omega}\left(u-\int_{\Omega} u d \gamma_{N}\right)^{2} d \gamma_{N} \leq \int_{\Omega}|D u|^{2} d \gamma_{N}, \quad \forall u \in H^{1}\left(\Omega, d \gamma_{N}\right) .
$$

It is well known (see, e.g., [20]) that, when $\Omega=\mathbb{R}^{N}$, inequality (1.3) still holds true. The purpose of this paper is to fill the gap between convex, bounded sets and the whole $\mathbb{R}^{N}$ by proving the following sharp lower bound.

Theorem 1.1. Let $\Omega \subset \mathbb{R}^{N}$ be a convex, $C^{2}$ domain whose boundary satisfies a uniform interior sphere condition (see (2.1) below). Then

$$
\mu_{1}(\Omega) \geq 1,
$$

equality holding if $\Omega$ is any $N$-dimensional strip.

Our strategy consists into constructing a suitable sequence $\left\{\Omega_{k}\right\}_{k \in \mathbb{N}}$ of bounded convex domains invading $\Omega$ and then passing to the limit in (1.2). To show that $\mu_{1}\left(\Omega_{k}\right)$ converge to $\mu_{1}(\Omega)$ one of the main ingredients is an extension theorem for functions belonging to $H^{1}\left(\Omega_{k}, d \gamma_{N}\right)$ with a constant independent of $k$ (see Theorem 2.1, see also [19]).

The structure of the differential operator in (1.1) suggests the relevance of the case of unbounded sets since the density of Gaussian measure degenerates at infinity. Moreover, in such a case, the space $H^{1}\left(\Omega, d \gamma_{N}\right)$ does no longer coincide with $H^{1}(\Omega)$. Indeed, when $\Omega=\mathbb{R}^{N}$, the case mostly studied by physicists, the eigenvalues of problem (1.1) are the integers and the corresponding eigenfunctions are combinations of Hermite polynomials, which clearly do not belong to $H^{1}\left(\mathbb{R}^{N}\right)$.

The Hermite operator appearing in problem (1.1) is widely studied in literature from many points of view. It is a classical subject in quantum mechanics (see, for instance, [20]) as well as in probability; indeed it is the generator of the OrnsteinUhlenbeck semigroup (see, e.g., [10]). Finally, problems of the kind (1.1) are related to some functional inequalities as the well-known Gross Theorem on the Sobolev Logarithmic embedding (see, e. g., $[11,21,24,27,28,32,40]$ ).

Note that the convexity assumption in Theorem 1.1 cannot be relaxed; it is enough to consider the classical example of a planar domain made by two equal squares connected by a thin corridor. Problems linking the geometry of a domain and the sequence of eigenvalues of a second-order elliptic operator are classical since the estimates by Faber, Krahn or Pólya, Szégö concerning the first eigenvalue of the Laplacian with Dirichlet or Neumann boundary conditions, respectively. Further developments of this topic can be found for instance in $[1,4,5,8,15-17,22,26,38]$, where estimates for Dirichlet eigenvalues and eigenfunctions of linear and nonlinear operators are derived. Concerning Neumann boundary conditions we refer the reader to $[5,12,14]$ and 
to $[6,18,29,30,39]$ for lower bounds of Neumann eigenvalues in different contexts (Laplacian, $p$-Laplacian, manifolds of constant curvature). For results in Gauss space we mention, for instance, $[9,13,23]$. Clearly the above list of references is far from being exhaustive; more papers in this growing field of research are cited in [33-36].

\section{Proof of Theorem 1.1}

We recall that, given a subset $\Omega$ of $\mathbb{R}^{N}$, $\partial \Omega$ satisfies a uniform interior sphere condition if

$$
\exists \bar{r}>0: \quad \forall x \in \partial \Omega \quad \exists B_{\bar{r}} \subset \Omega \text { such that } \overline{B_{\bar{r}}} \cap \bar{\Omega}=\{x\},
$$

where $B_{\bar{r}}$ denotes a ball with radius $\bar{r}>0$.

The proof of our result is divided in two steps. The first one provides an extension theorem that may have an interest by its own. In the second one, we consider a sequence of convex, bounded domains $\left\{\Omega_{k}\right\}_{k \in \mathbb{N}}$ invading $\Omega$ satisfying $\mu_{1}\left(\Omega_{k}\right) \geq 1$ and we show that $\lim _{k} \mu_{1}\left(\Omega_{k}\right)=\mu_{1}(\Omega)$.

Theorem 2.1. Let $\Omega \subset \mathbb{R}^{N}$ be a convex, $C^{2}$ domain whose boundary satisfies (2.1) and let us denote $d_{0}=\operatorname{dist}(0, \partial \Omega)$. Let $u \in H^{1}\left(\Omega, d \gamma_{N}\right)$; there exists a function $\tilde{u} \in H^{1}\left(\mathbb{R}^{N}, d \gamma_{N}\right)$ extending $u$ to the whole $\mathbb{R}^{N}$ and a constant $C$ such that

$$
\|\tilde{u}\|_{H^{1}\left(\mathbb{R}^{N}, d \gamma_{N}\right)} \leq C\|u\|_{H^{1}\left(\Omega, d \gamma_{N}\right)} .
$$

In (2.2) $C=C(\bar{r}, N)$ if $0 \in \Omega$, while $C=C\left(\bar{r}, N, d_{0}\right)$ if $0 \notin \Omega$.

Proof of Theorem 2.1. We distinguish two cases: $0 \in \Omega$ and $0 \notin \Omega$ and we fix $\tilde{r}=\frac{\bar{r}}{2}$.

Suppose first that $0 \in \Omega$. Let us denote by $d(x)=\operatorname{dist}(x, \partial \Omega)$ the distance of a point $x \in \mathbb{R}^{N}$ from $\partial \Omega$ and

$$
\Omega^{\tilde{r}}=\left\{x \in \mathbb{R}^{N} \backslash \bar{\Omega}: d(x)<\tilde{r}\right\}, \quad \Omega_{\tilde{r}}=\{x \in \Omega: d(x)<\tilde{r}\} .
$$

Let $u \in H^{1}\left(\Omega, d \gamma_{N}\right)$; we want to extend $u$ to $\mathbb{R}^{N}$ by reflection along the normal to $\partial \Omega$. Define

$$
\Phi: x \in \Omega_{\tilde{r}} \longrightarrow \Phi(x)=x-2 d(x) \operatorname{Dd}(x) \in \Omega^{\tilde{r}} .
$$

By construction $\Phi$ is a $C^{1}$ one-to-one map; we claim that

$$
1 \leq\left|J_{\Phi}(x)\right| \leq 3^{N-1}, \quad \forall x \in \Omega_{\tilde{r}} .
$$

A straightforward computation yields

$$
\frac{\partial \Phi_{i}(x)}{\partial x_{j}}=\delta_{i j}-2 \frac{\partial d(x)}{\partial x_{j}} \frac{\partial d(x)}{\partial x_{i}}-2 d(x) \frac{\partial^{2} d(x)}{\partial x_{i} \partial x_{j}} .
$$

By a rotation of coordinates we can assume that the $x_{N}$-axis lies in the direction $\operatorname{Dd}(x)$. By a further rotation of the first $N-1$ coordinates we can also assume that the $x_{1}, \ldots, x_{N-1}$ axes lie along the principal directions corresponding to the principal curvatures $\kappa_{1}, \ldots, \kappa_{N-1}$ of $\partial \Omega$ at $p(x)=\frac{x+\Phi(x)}{2}$. Clearly $p(x)$ is the projection of $x$ on $\partial \Omega$. In this coordinate system, known as principal coordinate system at $p(x)$, it is immediate to verify that

$$
\left|J_{\Phi}(x)\right|=\prod_{i=1}^{N-1}\left(1+\frac{2 d(x) \kappa_{i}}{1-d(x) \kappa_{i}}\right) .
$$

Claim (2.3) follows recalling that $d(x)<\tilde{r}=\frac{\bar{r}}{2}$ and $\kappa_{i} \leq \frac{1}{\bar{r}}$. 
We observe that in the simplest case $N=2,(2.3)$ has been proven, in a different and more direct way, in [13].

Now define

$$
\bar{u}(x)=u\left(\Phi^{-1}(x)\right) \quad \forall x \in \Omega^{\tilde{r}} .
$$

Let $\theta \in C_{0}^{\infty}\left(\mathbb{R}^{N}\right)$ be a cut-off function such that $0 \leq \theta \leq 1$ in $\mathbb{R}^{N}, \theta=1$ in $\Omega, \theta=0$ in $\mathbb{R}^{N} \backslash\left(\Omega \cup \Omega^{\tilde{r}}\right)$ and $|D \theta| \leq C=C(\bar{r})$. Set

$$
\tilde{u}= \begin{cases}u & \text { in } \Omega \\ \theta \bar{u} & \text { in } \Omega^{\tilde{r}} \\ 0 & \text { in } \mathbb{R}^{N} \backslash\left(\Omega \cup \Omega^{\tilde{r}}\right) .\end{cases}
$$

Since $\Omega$ contains the origin, it is easy to verify that

$$
\exp \left(\frac{-|\Phi(x)|^{2}}{2}+\frac{|x|^{2}}{2}\right) \leq 1, \quad \forall x \in \Omega_{\tilde{r}}
$$

Thus, by (2.3), (2.4) and (2.5) we get

$$
\begin{aligned}
\int_{\mathbb{R}^{N}} \tilde{u}^{2} d \gamma_{N} & =\int_{\Omega} u^{2} d \gamma_{N}+\int_{\Omega^{\tilde{r}}} \tilde{u}^{2} d \gamma_{N} \\
& \leq \int_{\Omega} u^{2} d \gamma_{N}+\int_{\Omega_{\tilde{r}}} u^{2}(x) \exp \left(-\frac{|\Phi(x)|^{2}}{2}+\frac{|x|^{2}}{2}\right)\left|J_{\Phi}\right| d \gamma_{N} \\
& \leq C(N) \int_{\Omega} u^{2} d \gamma_{N} .
\end{aligned}
$$

On the other hand, (2.3), (2.4), (2.5) and (2.6) imply

$$
\begin{aligned}
& \int_{\mathbb{R}^{N}}|D \tilde{u}|^{2} d \gamma_{N} \leq C(N, \bar{r})\left[\int_{\Omega^{\tilde{r}}} \bar{u}^{2} d \gamma_{N}+\int_{\Omega \cup \Omega^{\tilde{r}}}|D \bar{u}|^{2} d \gamma_{N}\right] \\
& \quad \leq C(N, \bar{r})\left[\int_{\Omega} u^{2} d \gamma_{N}+\int_{\Omega}|D u|^{2} d \gamma_{N}+\int_{\Omega_{\tilde{r}}}|D u|^{2} \exp \left(-\frac{|\Phi(x)|^{2}}{2}+\frac{|x|^{2}}{2}\right)\left|J_{\Phi}\right| d \gamma_{N}\right] \\
& \quad \leq C(N, \bar{r})\left[\int_{\Omega} u^{2} d \gamma_{N}+\int_{\Omega}|D u|^{2} d \gamma_{N}\right]
\end{aligned}
$$

Hence, if $\Omega$ contains the origin (2.2) holds true.

Suppose now that $0 \notin \Omega$. Up to a rotation about the origin, the translation $T$ : $x=\left(x_{1}, x_{2}, \ldots, x_{N}\right) \in \mathbb{R}^{N} \rightarrow\left(x_{1}-\delta, x_{2}, \ldots, x_{N}\right) \in \mathbb{R}^{N}$, for a fixed $\delta>d_{0}$, maps $\Omega$ onto a set $T(\Omega)$ containing the origin. Define

$$
v(x)=v\left(x_{1}, x_{2}, \ldots, x_{N}\right)=u\left(x_{1}+\delta, x_{2}, \ldots, x_{N}\right) \exp \left(-\frac{x_{1} \delta}{2}-\frac{\delta^{2}}{4}\right), \quad x \in T(\Omega)
$$


then

$$
\int_{\Omega} u^{2} d \gamma_{N}=\int_{T(\Omega)} v^{2} d \gamma_{N}
$$

By construction $T(\Omega)$ contains the origin, then there exists a function $\tilde{v} \in H^{1}\left(\mathbb{R}^{N}, d \gamma_{N}\right)$ such that $\left.\tilde{v}\right|_{T(\Omega)}=v$ and

$$
\|\tilde{v}\|_{H^{1}\left(\mathbb{R}^{N}, d \gamma_{N}\right)} \leq C(\bar{r}, N)\|v\|_{H^{1}\left(T(\Omega), d \gamma_{N}\right)} .
$$

Let

$$
\tilde{u}(x)=\tilde{u}\left(x_{1}, x_{2}, \ldots, x_{N}\right)=\tilde{v}\left(x_{1}-\delta, x_{2}, \ldots, x_{N}\right) \exp \left(\frac{x_{1} \delta}{2}-\frac{\delta^{2}}{4}\right),
$$

we finally get

$$
\|\tilde{u}\|_{H^{1}\left(\mathbb{R}^{N}, d \gamma_{N}\right)} \leq C\left(\bar{r}, N, d_{0}\right)\|u\|_{H^{1}\left(\Omega, d \gamma_{N}\right)} .
$$

Using the fact that $H^{1}\left(\mathbb{R}^{N}, d \gamma_{N}\right)$ is compactly embedded into $L^{2}\left(\mathbb{R}^{N}, d \gamma_{N}\right)$ (see, e.g. [25]) and the above extension theorem we deduce the compact embedding of $H^{1}\left(\Omega, d \gamma_{N}\right)$ into $L^{2}\left(\Omega, d \gamma_{N}\right)$. By classical arguments (see, for instance, Theorem 5.4.3 in [7], see also Proposition 3.4 in [31] we can deduce a Poincaré-Wirtinger inequality with a constant depending on $\Omega$. Therefore, as we said in Section 1 , by the classical spectral theory on compact self-adjoint operators, $\mu_{1}(\Omega)$ satisfies the following variational characterization

$$
\mu_{1}(\Omega)=\min \left\{\frac{\int_{\Omega}|D \psi|^{2} d \gamma_{N}}{\int_{\Omega}|\psi|^{2} d \gamma_{N}}: \psi \in H^{1}\left(\Omega, d \gamma_{N}\right) \backslash\{0\}, \int_{\Omega} \psi d \gamma_{N}=0\right\}
$$

When $\Omega$ is a convex, bounded domain, estimate (1.4) is contained in [3]. Therefore, from now on $\Omega$ will denote a convex, unbounded domain. Let $\left\{\Omega_{k}\right\}_{k \in \mathbb{N}}$ be a sequence of convex, bounded, $C^{2}$ domains whose boundaries satisfy (2.1) for every $k \in \mathbb{N}$, and invading $\Omega$ in the sense that

$$
\Omega_{k} \subset \Omega_{k+1} \quad \forall k \in \mathbb{N} \text { and } \bigcup_{k \in \mathbb{N}} \Omega_{k}=\Omega
$$

For the explicit construction of a sequence of this kind, see for instance [13].

As proven in [3] we have that

$$
\mu_{1}\left(\Omega_{k}\right) \geq 1, \quad \forall k \in \mathbb{N}
$$

that can be equivalently written as

$$
\int_{\Omega_{k}} \psi^{2} d \gamma_{N} \leq \int_{\Omega_{k}}|D \psi|^{2} d \gamma_{N}, \quad \forall \psi \in H^{1}\left(\Omega_{k}, d \gamma_{N}\right): \int_{\Omega_{k}} \psi d \gamma_{N}=0 .
$$

Now we want to pass to the limit in (2.7). To this aim consider the operator

$$
A_{k}: f \in L^{2}\left(\Omega, d \gamma_{N}\right): \int_{\Omega} f d \gamma_{N}=0 \longrightarrow \tilde{u}_{k} \in H^{1}\left(\Omega, d \gamma_{N}\right),
$$


where $\tilde{u}_{k}$ is the extension provided in Theorem 2.1 of the solution $u_{k} \in H^{1}\left(\Omega_{k}, d \gamma_{N}\right)$ to the following problem:

$$
\begin{cases}-\operatorname{div}\left(\exp \left(-\frac{|x|^{2}}{2}\right) D u_{k}\right)=\left(f-c_{k}\right) \exp \left(-\frac{|x|^{2}}{2}\right) & \text { in } \Omega_{k} \\ \frac{\partial u_{k}}{\partial \nu_{k}}=0 & \text { on } \partial \Omega_{k} \\ \int_{\Omega_{k}} u_{k} d \gamma_{N}=0 & \end{cases}
$$

where $c_{k}=\int_{\Omega_{k}} f d \gamma_{N}$ and $\nu_{k}$ is the outward normal to $\partial \Omega_{k}$. Observe that LaxMilgram theorem ensures the existence and uniqueness of $u_{k}$. Moreover, $\gamma_{N}\left(\Omega \backslash \Omega_{k}\right) \rightarrow$ 0 implies $c_{k} \rightarrow 0$. We also introduce the operator

$$
A: f \in L^{2}\left(\Omega, d \gamma_{N}\right): \int_{\Omega} f d \gamma_{N}=0 \longrightarrow u \in H^{1}\left(\Omega, d \gamma_{N}\right)
$$

where $u$ is the unique solution, whose existence is guaranteed by Lax-Milgram theorem, to the problem

$$
\begin{cases}-\operatorname{div}\left(\exp \left(-\frac{|x|^{2}}{2}\right) D u\right)=f \exp \left(-\frac{|x|^{2}}{2}\right) & \text { in } \Omega \\ \frac{\partial u}{\partial \nu}=0 & \text { on } \partial \Omega \\ \int_{\Omega} u d \gamma_{N}=0 . & \end{cases}
$$

Using $u_{k}$ as test function in (2.9), from Schwarz inequality we deduce

$$
\int_{\Omega_{k}}\left|D u_{k}\right|^{2} d \gamma_{N}=\int_{\Omega_{k}} u_{k}\left(f-c_{k}\right) d \gamma_{N} \leq\left(\int_{\Omega_{k}} u_{k}^{2} d \gamma_{N}\right)^{1 / 2}\left(\int_{\Omega_{k}}\left(f-c_{k}\right)^{2} d \gamma_{N}\right)^{1 / 2} .
$$

Using (2.8) and recalling that $c_{k} \rightarrow 0$, we get

$$
\int_{\Omega_{k}}\left|D u_{k}\right|^{2} d \gamma_{N} \leq C_{1} \int_{\Omega} f^{2} d \gamma_{N}+C_{2},
$$

where $C_{1}, C_{2}$ are positive constants whose values are independent of $k$. The above inequality together with (2.8) yield

$$
\int_{\Omega_{k}} u_{k}^{2} d \gamma_{N}+\int_{\Omega_{k}}\left|D u_{k}\right|^{2} d \gamma_{N} \leq C
$$

where $C$ is a positive constant whose value is independent of $k$. From (2.2) we deduce that the sequence $\left\{\tilde{u}_{k}\right\}_{k \in \mathbb{N}}$ is bounded in $H^{1}\left(\Omega, d \gamma_{N}\right)$. Since the embedding of $H^{1}\left(\Omega, d \gamma_{N}\right)$ into $L^{2}\left(\Omega, d \gamma_{N}\right)$ is compact, there exists a (not relabelled) subsequence $\left\{\tilde{u}_{k}\right\}_{k \in \mathbb{N}}$ such that $\tilde{u}_{k} \rightarrow v$ in $H^{1}\left(\Omega, d \gamma_{N}\right), \tilde{u}_{k} \rightarrow v$ in $L^{2}\left(\Omega, d \gamma_{N}\right)$ and a.e. in $\Omega$. In fact, $v$ coincides with $u$ since they both solve the same problem (2.10). Indeed, let 
$\phi \in C^{\infty}(\Omega)$. Recalling that $\gamma_{N}\left(\Omega \backslash \Omega_{k}\right) \rightarrow 0$ and $\Omega_{k} \subset \Omega$, we get

$$
\begin{aligned}
\int_{\Omega} D v D \phi d \gamma_{N} & =\lim _{k} \int_{\Omega} D \tilde{u}_{k} D \phi d \gamma_{N}=\lim _{k}\left(\int_{\Omega_{k}} D u_{k} D \phi d \gamma_{N}+\int_{\Omega \backslash \Omega_{k}} D \tilde{u}_{k} D \phi d \gamma_{N}\right) \\
& =\lim _{k} \int_{\Omega_{k}}\left(f-c_{k}\right) \phi d \gamma_{N}=\int_{\Omega} f \phi d \gamma_{N} .
\end{aligned}
$$

Finally, as $k$ goes to $+\infty$,

$$
\left\|\left(A_{k}-A\right) f\right\|_{L^{2}\left(\Omega, d \gamma_{N}\right)}=\left\|\tilde{u}_{k}-u\right\|_{L^{2}\left(\Omega, d \gamma_{N}\right)} \rightarrow 0 .
$$

The compact embedding of $H^{1}\left(\Omega, d \gamma_{N}\right)$ into $L^{2}\left(\Omega, d \gamma_{N}\right)$ and (2.11) allow us to adapt Theorems 2.3.1 and 2.3.2 in [33] to conclude that the operators $A_{k}$ strongly converge to $A$ and hence

$$
\mu_{1}\left(\Omega_{k}\right) \rightarrow \mu_{1}(\Omega)
$$

Finally, we prove the optimality of our estimate (1.4). Consider the $N$-dimensional strip

$$
S_{a}=\left\{x=\left(x_{1}, x_{2}, \ldots, x_{N}\right) \in \mathbb{R}^{N}:-a<x_{1}<a, x_{2}, \ldots, x_{N} \in \mathbb{R}\right\}, \quad a \in(0,+\infty) .
$$

The eigenfunctions are factorized and can be written as linear combinations of products of homogeneous Hermite polynomials $H_{m_{1}}\left(x_{1}\right), H_{m_{2}}\left(x_{2}\right), \ldots, H_{m_{N}}\left(x_{N}\right)$. We recall that the Hermite polynomials in one variable are defined by

$$
H_{m}(t)=(-1)^{m} e^{t^{2} / 2} \frac{d^{m}}{d t^{m}} e^{-t^{2} / 2}, \quad m \in \mathbb{N} \cup\{0\},
$$

and they constitute a complete set of eigenfunctions to problem (1.1) when $\Omega=\mathbb{R}$; more precisely

$$
-\left(e^{-t^{2} / 2} H_{m}^{\prime}(t)\right)^{\prime}=m e^{-t^{2} / 2} H_{m}(t), \quad m \in \mathbb{N} \cup\{0\} .
$$

Denote by $\lambda_{1}(-a, a)$ the first Dirichlet eigenvalue of the one-dimensional Hermite operator in the interval $(-a, a)$. One can easily verify that

$$
\mu_{1}(-a, a)=\lambda_{1}(-a, a)+1>1=\mu_{1}(\mathbb{R}) .
$$

Therefore, $\mu_{1}\left(S_{a}\right)=1$ for every $a \in(0,+\infty)$ and a corresponding eigenfunction is, for instance, $H_{1}\left(x_{2}\right)=x_{2}$.

\section{References}

[1] A. Alvino, V. Ferone and G. Trombetti, On the properties of some nonlinear eigenvalues, SIAM J. Math. Anal. 29(2) (1998), 437-451.

[2] B. Andrews and J. Clutterbuck, Proof of the fundamental gap conjecture, J. Amer. Math. Soc. 24(3) (2011), 899-916.

[3] B. Andrews and L. Ni, Eigenvalue comparison on Bakry-Emery manifolds, Commun. Partial Differ. Equ. 37 (2012), 2081-2092.

[4] M.S. Ashbaugh and R.D. Benguria, A sharp bound for the ratio of the first two eigenvalues of Dirichlet Laplacians and extensions, Ann. Math. (2) 135(3) (1992), 601-628.

[5] M.S. Ashbaugh and R.D. Benguria, Sharp upper bound to the first nonzero Neumann eigenvalue for bounded domains in spaces of constant curvature., J. Lond. Math. Soc. (2) 52(2) (1995), $402-416$.

[6] M.S. Ashbaugh and L. Hermi, On extending the inequalities of Payne, Pólya, and Weinberger using spherical harmonics, Rocky Mountain J. Math. 38(4) (2008), 1037-1072. 
[7] H. Attouch, G. Buttazzo and G. Michaille, Variational analysis in Sobolev and BV spaces, Applications to PDEs and optimization. MPS/SIAM Series on Optimization, 6, SIAM, 2006.

[8] R.D. Benguria, H. Linde and B. Loewe, Isoperimetric inequalities for eigenvalues of the Laplacian and the Schrödinger operator, Bulletin of Mathematical Sciences 2 (2012), 1-56.

[9] M.F. Betta, F. Chiacchio and A. Ferone, Isoperimetric estimates for the first eigenfunction of a class of linear elliptic problems, Z. Angew. Math. Phys. 58(1) (2007), 37-52.

[10] V.I. Bogachev, Gaussian Measures, Mathematical Surveys and Monographs, 62, American Mathematical Society, Providence, RI, 1998.

[11] B. Brandolini, F. Chiacchio and C. Trombetti, Hardy type inequalities and Gaussian measure, Commun. Pure Appl. Anal. 6(2) (2007), 411-428.

[12] B. Brandolini, F. Chiacchio and C. Trombetti, Sharp estimates for eigenfunctions of a Neumann problem, Comm. Partial Differ. Equ. 34(10-12) (2009), 1317-1337.

[13] B. Brandolini, F. Chiacchio and C. Trombetti, A sharp lower bound for some Neumann eigenvalues of the Hermite operator, Differ. Integral Eq. 26(5-6) (2013), 639-654.

[14] B. Brandolini, F. Chiacchio and C. Trombetti, Optimal lower bounds for eigenvalues of linear and nonlinear Neumann problems, Proc. Roy. Soc. Edinburgh Sect. A., to appear.

[15] B. Brandolini, P. Freitas, C. Nitsch and C. Trombetti, Sharp estimates and saturation phenomena for a nonlocal eigenvalue problem, Adv. Math. 228(4) (2011), 2352-2365.

[16] B. Brandolini, C. Nitsch and C. Trombetti, New isoperimetric estimates for solutions to MongeAmpère equations, Ann. Inst. H. Poincaré Anal. Non Linéaire 26(4) (2009), 1265-1275.

[17] B. Brandolini, C. Nitsch and C. Trombetti, An upper bound for nonlinear eigenvalues on convex domains by means of the isoperimetric deficit, Arch. Math. (Basel) 94(4) (2010), 391-400.

[18] F. Brock, F. Chiacchio and A. Mercaldo, Weighted isoperimetric inequalities in cones and applications, Nonlinear Anal. 75(15) (2012), 5737- 5755.

[19] D. Chenais, On the existence of a solution in a domain identification problem, J. Math. Anal. Appl. 52 (1975), 189-219.

[20] R. Courant and D. Hilbert, Methods of mathematical physics. Vol. I., Interscience Publishers, Inc., New York, N.Y., 1953.

[21] F. Chiacchio, Comparison results for linear parabolic equations in unbounded domains via Gaussian symmetrization, Differential and Integral Equations 17 (2004), 241-258.

[22] F. Chiacchio, Estimates for the first eigenfunction of linear eigenvalue problems via Steiner symmetrization, Publ. Mat. 53(1) (2009), 47-71.

[23] F. Chiacchio and G. Di Blasio, Isoperimetric inequalities for the first Neumann eigenvalue in Gauss space, Ann. Inst. H. Poincaré Anal. Non Linéaire 29(2) (2012), 199-216.

[24] A. Cianchi and L. Pick, Optimal Gaussian Sobolev embeddings, J. Funct. Anal. 256(11) (2009), 3588-3642.

[25] G. Da Prato, An introduction to infinite-dimensional analysis. Revised and extended from the 2001 original by Da Prato. Universitext. Springer-Verlag, Berlin, 2006.

[26] F. Della Pietra and N. Gavitone, Sharp bounds for the first eigenvalue and the torsional rigidity related to some anisotropic operators, Math. Nachr. (2013), DOI:10.1002/mana.201200296.

[27] A. Ehrhard, Inégalités isopérimetriques et intégrales de Dirichlet Gaussiennes, Ann. Sci. Ecole Norm. Supl. 17 (1984), 317-332.

[28] A. Ehrhard, Éléments extrémaux pour les inégalités de Brunn-Minkowski Gaussiennes, Ann. Inst. H. Poincaré Probab. Statist. 22(2) (1986), 149-168.

[29] L. Esposito, C. Nitsch and C. Trombetti, Best constants in Poincaré inequalities for convex domains, J. Convex Anal. 20(1) (2013), 253-264.

[30] V. Ferone, C. Nitsch and C. Trombetti, A remark on optimal weighted Poincaré inequalities for convex domains, Rendiconti Lincei Matematica e Applicazioni 23(4) (2012), 467-475.

[31] F. Feo and M. R. Posteraro, Logarithmic Sobolev trace inequalities, Asian J. Math., 17(3) (2013), 569-582.

[32] L. Gross, Logarithmic Sobolev inequalities, Amer. J. Math. 97(4) (1975), 1061-1083.

[33] A. Henrot, Extremum problems for eigenvalues of elliptic operators, Frontiers in Mathematics. Birkhäuser Verlag, Basel, 2006.

[34] A. Henrot and M. Pierre, Variation et optimisation de formes. Une analyse géométrique, Mathematics \& Applications, 48. Springer, Berlin, 2005. 
[35] B. Kawohl, Rearrangements and convexity of level sets in PDE. Lecture Notes in Mathematics, 1150. Springer-Verlag, Berlin, 1985.

[36] S. Kesavan, Symmetrization \& applications. Series in Analysis, 3. World Scientific Publishing Co. Pte. Ltd., Hackensack, NJ, 2006.

[37] Lei Ni, Estimates on the modulus of expansion for vector fields solving nonlinear equations, J. Math. Pures Appl. 99(1) (2013), 1-16.

[38] C. Nitsch, An isoperimetric result for the fundamental frequency via domain derivative, Calc. Var. Partial Differential Equations (2012), DOI:10.1007/s00526-012-0584-2.

[39] L.E. Payne and H.F. Weinberger, An optimal Poincaré inequality for convex domains, Arch. Rational Mech. Anal. 5 (1960), 286-292.

[40] E. Pelliccia and G. Talenti, A proof of a logarithmic Sobolev inequality, Calc. Var. Partial Differ. Equ. 1(3) (1993), 237-242.

Dipartimento di Matematica e Applicazioni "R. Caccioppoli", Università degli Studi di Napoli "Federico II", Complesso Monte S. Angelo, via Cintia - 80126, Napoli, Italy

E-mail address: brandolini@unina.it

Dipartimento di Matematica e Applicazioni "R. Caccioppoli", Università degli Studi Di Napoli "Federico II", Complesso Monte S. Angelo, via Cintia - 80126 Napoli, Italy

E-mail address: fchiacch@unina.it

Institut Elie Cartan, UMR CNRS 7502, Université de Lorraine, F-54506 VandoeuvreLes-Nancy Cedex, France

E-mail address: antoine.henrot@univ-lorraine.fr

Dipartimento di Matematica e Applicazioni "R. Caccioppoli", Università degli Studi di Napoli "Federico II", Complesso Monte S. Angelo, via Cintia - 80126 Napoli, Italy

E-mail address: cristina@unina.it 
